\title{
Du charme des petits congrès
}

\section{Dominik Heim}

PD Dr méd., ancien médecin-chef de chirurgie, Hôpital Frutigen, membre d’honneur de la Société suisse de chirurgie et de la 'European Society for Trauma and Emergency Surgery', membre de la FMH

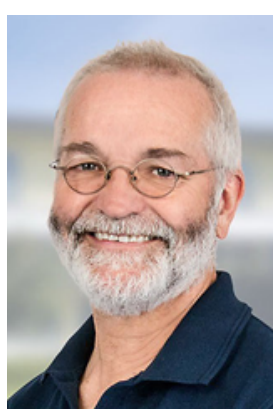

Il est clair que les petits ont du mal à s'en sortir! Le mail a été envoyé à cent vingt et un membres: «Si nous ne remplissons pas l'hôtel, le congrès sera annulé.» Ce serait vraiment dommage, parce qu'il a lieu à Roscoff, au bord de la Manche. Là où le vent souffle en permanence, où poussent les artichauts et où le commissaire Dupin doit résoudre ses affaires insolites [1]. Ce Parisien en exil n'arriverait à rien sans Nolweyn, sa secrétaire locale qui, grâce à sa connaissance des légendes et du contexte breton, lui apporte l'aide dont il a besoin pour démêler, au terme de nombreuses pages de fiction, l'écheveau complexe de l'énigme. Petite remarque en passant: dans le milieu médical, lorsque l'on n'est pas du coin, on a besoin de quelqu'un qui l'est. Si précise que soit une anamnèse, elle ne renseigne pas toujours d'emblée sur les conditions psycho-socio-économiques, alors que l'assistante médicale locale le peut! Mais «on n'a pas toujours un Dupin», allusion discrète $\mathrm{au}$ livre culte des années 60 sur le caviar, signé Johannes Mario Simmel [2] - oui, oui, je sais, toujours ces renvois à la culture d'autrefois, que les jeunes lecteurs ne peuvent pas connaître! Mais on peut bien parsemer un article de quelques gourmandises littéraires, non? Et il n'y a certainement rien de mal à avoir des connaissances sur le passé, cela fait partie d'une bonne culture générale.

L'histoire du congrès près de la Manche n'est pas un exemple isolé. Dans la même veine, il y a eu le congrès SITEMSH [3], dont les participants ont vanté l'excellence scientifique. Mais ils ont en réalité été peu nombreux à en profiter. Sans l'engagement désintéressé des secrétaires nationaux, qui, traditionnellement, paient leurs frais de congrès même lorsqu'ils interviennent comme conférenciers, la manifestation aurait été un désastre financier. Leur bonne volonté permet à la société de continuer d'exister. Et il s'agit sans doute de la plus importante et compétente des sociétés de médecine des sports d'hiver.

Dans notre pays aussi, il existe de nombreuses petites sociétés dont le thème devrait intéresser un vaste public et qui luttent pourtant pour survivre: les journées biennoises de formation continue (www.sgact.ch) se sont développées traditionnellement, leur mission est la chirurgie générale, qui conserve une grande place, du moins en milieu rural (en agglomération, le centre hospitalier spécialisé est devenu «la référence»). Le nombre de participants diminue, ce qui incite l'industrie à ne plus s'impliquer et, lorsque les chiffres sont dans le rouge, une mission perd ses moyens d'existence. Oncle Picsou règne sur la médecine depuis longtemps déjà. Le nombre de cas est le pilier de l'économicité, la qualité technique risque de partir en fumée - vous savez, que dans $40 \%$ environ des dossiers soumis pour examen, la réponse à la question de l'existence d'une erreur thérapeutique est aujourd'hui affirmative!

Les petits congrès vivent de l'engagement de leurs promoteurs et d'une industrie raisonnable. Ils permettent un contact personnel entre les participants et avec la faculté - c'est ce que l'on appelle aujourd'hui le réseautage. La priorité y est donnée à la participation active; il est presque impossible de s'y noyer dans la masse. Et c'est moins stérile que de télécharger les PDF des conférences depuis le Net. J'affirme que l'efficacité en termes d'apprentissage est bien plus grande qu'avec l'e-learning ou lors des méga-congrès comptant des milliers de participants. Si l'on n'est pas timide, on se rend aux petits congrès où l'on rencontre les participants et les membres de la faculté sur un pied d'égalité et l'on se fait expliquer en personne les problèmes et les possibles solutions.

Nous nous verrons aux journées biennoises de formation continue, les 6 et 7 septembre 2018. Elles proposent aussi de la culture, ce qui, en médecine, est presque devenu inhabituel.

P.-S.: Je ne veux pas priver le lecteur bienveillant de cette annonce parue dans le journal SonntagsZeitung: "C'est chez le boulanger qu'on trouve le meilleur pain. Autrement dit, chez nous.» Elle est signée Migros! On ne saurait mieux résumer la mondialisation! Et cela cadre si bien avec ce qui précède...

\section{Crédit photo \\ (c) Hohmad}

\section{Références}

1 Bannalec JL. Un été à Pont-Aven. Presses de la Cité; 2014.

2 Simmel JM. On n'a pas toujours du caviar. Robert Laffont; 1970.

3 Heim D. Prevention is better than healing. Bull Méd Suisses. 2018;99(6):188-90. 